\title{
Experimental and Numerical Studies of Vortex Induced Vibration on Cylinder
}

\author{
M. Mobassher Tofaa,b, Adi Maimun ${ }^{b^{*}}$, Yasser M. Ahmeda, Saeed Jameia, Hassan Abyn ${ }^{a}$ \\ ${ }^{a}$ Faculty of Mechanical Engineering, Universiti Teknologi Malaysia, 81310 UTM Johor Bahru, Johor, Malaysia \\ ${ }^{b}$ Marine Technology Centre, Universiti Teknologi Malaysia, 81310 UTM, Johor Bahru, Johor, Malaysia \\ *Corresponding author: adi@fkm.utm.my
}

\section{Article history}

Received :1 August 2013

Received in revised form :

10 November 2013

Accepted :28 November 2013

Graphical abstract

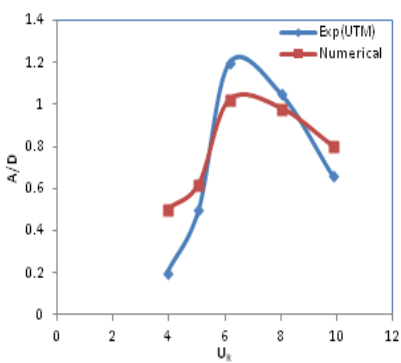

\section{Abstract}

Study of vibrations due to vortex shedding (VIV) in the wake of a cylinder that is exposed to current or oscillatory flow is very important, especially for marine risers which are used to extract oil and gas from the sea bed. The phenomenon of vortex induced vibration (VIV) has been one of the major concerns for hydrodynamic researchers due to its potential ability to cause severe fatigue damage. The hydrodynamics of VIV is very complex and still not fully understood. In this paper, some results (amplitude over diameter, lift and drag coefficients) of high Reynolds VIV experiments that are performed in UTM towing tank with an in-house test set-up are presented. A circular cylinder of $114 \mathrm{~mm}$ in diameter and 3 min length was towed at constant speed through the basin at Reynolds numbers up to $1.1 \times 10^{5}$. Model tests with a stationary cylinder and tests with a freely vibrating cylinder were carried out to investigate the influence of VIV on drag coefficient. Later these results are compared with results obtained through 3D numerical simulation, LES is used to solve turbulence flow. It was found that CFD results showed similar trends with experimental results. Results of this paper can be very important to design riser system and future endeavor to perform similar kind of experiments. Successful numerical study of the VIV can also be fruitful for designing efficient VIV suppression devices.

Keywords: VIV test setup; Large Eddy Simulation(LES)

(C) 2014 Penerbit UTM Press. All rights reserved.

\subsection{INTRODUCTION}

Vortex-induced vibration (VIV) is one of the key concerns for engineers while designing riser systems used for extracting oil and gas from the sea bed. A high level of fatigue damage can be occurred in a relatively short period of time for risers that are exposed to harsh ocean environments because of VIV. VortexInduced Vibration (VIV) is a phenomenon that happens when flow interacts with a certain structure as shown in Figure 1. When a fluid flows past a blunt object, it is excited by forces caused by vortices shed, an unsteady flow that occurs in special flow velocities depending upon the size and shape of the body. Vortices are created at the back of the body and separate periodically as well as asymmetrically from either side of the body causing the time varying non-uniform pressure distribution around the object. This non uniform pressure creates a time varying lift force around the object resulting the structure to vibrate in both inline and transverse to the flow. The vortex shedding frequency harmonizes with the natural frequency, and the vibration frequency near the natural frequency of the structure. The change in hydrodynamic mass is one of the main reasons behind this synchronization, as demonstrated in the experiments of Vikestad [1]. This synchronization occurs over a range of reduced velocity that is defined as the lock-in range. VIV is considered as a self-limiting type of response, usually after reaching certain amplitude shedding process no longer transfer the energy from fluid to structure, instead energy transfer process become reverse. Typically, the resulting vibrations are detrimental because it increases fatigue loading and component design complexity to accommodate these motions.

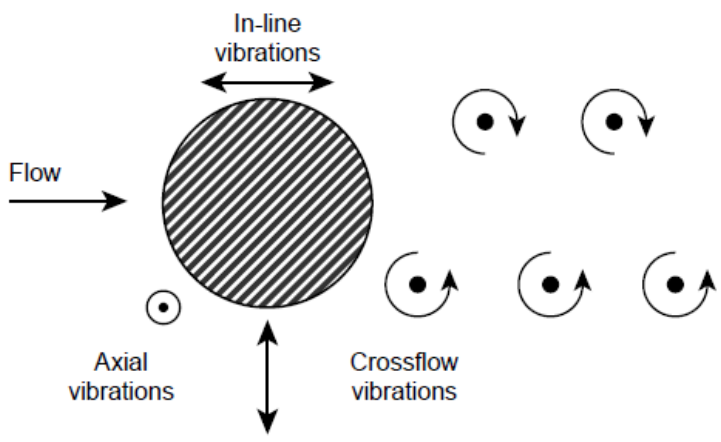

Figure 1 vortex induced vibration of cylinder 
Numerous experimental and numerical investigations, to study vortex-induced vibrations (VIV) of a circular cylinder, have been carried out in the recent past. In steady flow, the vortex shedding process is characterized by Reynolds number, the vortex shedding process remains more or less same for $300<\operatorname{Re}<300000$. This range of Reynolds number is termed as sub-critical flow regime, Though full scale cases certainly enter the critical and super-critical regimes where $\mathrm{Re}>300000$, most VIV related experiments had been done in sub-critical flow regime. Some of the initial research works on flow induced vibrations were summed up by Blevins [2]. It was found by numerous studies [2] that the in-line vibrations magnitude are smaller than the transverse vibrations. Inline vibrations are less important for risers since they only emerge at lower reduced velocity. A number of experimental evaluations of vortex formation modes were reviewed by Govardhan and Williamson [3]. Willden and Graham [4] reviewed VIV studies on low mass ratio cylinders. Dong and Karniadakis [5] research presents some VIV results of Direct Numerical Simulation (DNS) of the flow past a stationary cylinder and a vibrating cylinder using a spectral element method at $\mathrm{Re}=10000$. Dong et al. [6] showed similar DNS simulations and PIV measurements of the flow past a stationary cylinder at Re $=4000$ and 10000. Although results of the simulations are successful in terms of matching with the experiments, it was found that the use of the spectral element method to perform DNS required large computational resources. Chaplin et al. [7] reviewed and compared some existing CFD codes for riser VIV analysis. Experimental details for a long riser under a variety of current conditions were presented by Trim et al. [8]. A number of research works on complex modes were reviewed by Lucor et al. [9]. Holmes et al. [10] used a fully 3D simulation approach to analyze riser VIV and the effect of strakes Flow over a sphere by using Detached-Eddy Simulation was discussed in details by Constantinescu et al. in both subcritical [11] and super critical region [12]. Saltara et al. did a 3D simulation to analyze VIV by using Detached-Eddy Simulation for a Reynolds number $10^{4}$, his simulation showed good agreement with existing results [13]. Research related 3D simulation to analyze VIV can be very rewarding as successful simulation can be used to verify the effectiveness of new design of VIV suppression devices. Very small number of literatures regarding 3D VIV simulation can be found, most of the existing literatures about VIV simulations are $2 \mathrm{D}$ and performed at low Re numbers. In this paper, the results of VIV experiments that were performed in UTM MARIN's towing tank are presented. This research study was primarily done to obtain new VIV data of a cylinder with low mass ratio at high Reynolds numbers that can be used as validation material for CFD calculations and to develop a better test set-up for future VIV measurements. Tests with a stationary cylinder as well as with the cylinder freely vibrating were carried out to find the effect of VIV on in-line drag. The present VIV data of this research has been compared with results obtained through computational fluid dynamics by using commercial software ANSYS CFX, LES was used as the turbulence model.

\subsection{IMPORTANT PARAMETERS FOR VIV ANALYSIS}

The hydrodynamics of a fixed cylinder with diameter $\mathrm{D}$ in constant flow with velocity $\mathrm{U}$ is characterized by three parameters namely Reynolds number $\left(\mathrm{R}_{\mathrm{e}}\right)$, Strouhal number $(\mathrm{St})$ and reduced velocity $\left(U_{R}\right)$.

$\operatorname{Re}=\frac{\mathrm{UD}}{v}$ where $v$ is the viscosity of water.

$\mathrm{St}=\frac{\mathrm{Df}_{\mathrm{S}}}{\mathrm{U}}$

where $f_{s}$ shedding frequency,

Reduced velocity $\left(\mathrm{U}_{\mathrm{R}}\right)$ is defined by following equation

$\mathrm{U}_{\mathrm{R}}=\frac{\mathrm{U}}{\mathrm{f}_{\mathrm{n}} \mathrm{D}}$

where $f_{n}$ natural frequency,

Vortex shedding can be defined as a sinusoidal process, so we can find transverse force responsible for cross flow vibration which is in $\mathrm{z}$ direction as depicted in Figure 2 of a cylinder of length $\mathrm{L}$ and diameter $\mathrm{D}$ by following Equations [2],

$F_{L}=C_{L} 0.5 \rho L D U^{2} \sin \left(2 \pi \mathrm{f}_{\mathrm{s}} \mathrm{t}\right)$

Where

$F_{L}=$ lift force

$C_{L}=$ lift coefficient

$\mathrm{f}_{\mathrm{s}}=$ Vortex shedding frequency

$\rho=$ density of water

$\mathrm{L}=$ Length of the cylinder

The motion of the cylinder of mass $(\mathrm{m})$ and added mass $(\mathrm{m} 0)$ can be found by solving following second order differential equation

$\left(\mathrm{m}+\mathrm{m}_{0}\right) \ddot{\mathrm{z}}+2 \mathrm{~m} \zeta \omega_{z} \dot{z}+\mathrm{kz}=\mathrm{F}_{\mathrm{L}}$

Where,

$\zeta=$ damping factor of the cylinder

$\omega_{z}=$ cylinder circular natural frequency

$\mathrm{k}=$ spring constant

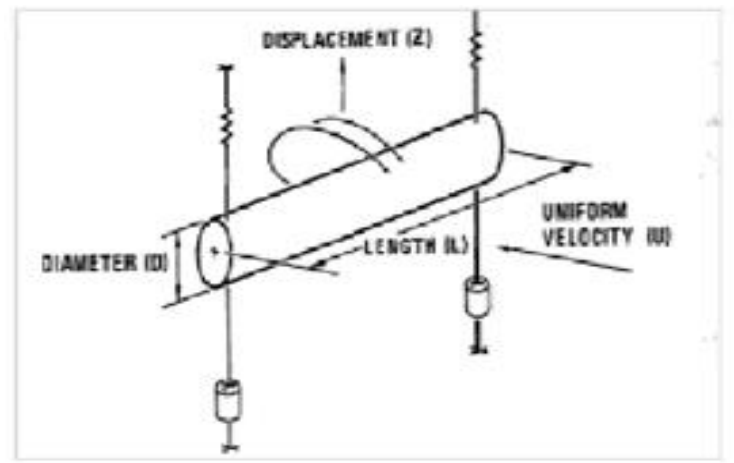

Figure 2 Cylinder and coordinate system [2]

The time varying lift force on the oscillating cylinder may have a phase $(\Phi)$ difference with the cylinder motion so solution of equation 4 can be written as

$z=A_{z} \sin \left(2 \pi \mathrm{f}_{\mathrm{s}} \mathrm{t}+\Phi\right)$

Where

$A_{Z}=$ maximum motion amplitude .and 


\section{$\Phi=$ Phase difference}

Drag of the cylinder can be directly measured with strain gauges on the blade springs, accelerometer is used to measure the vertical accelerations and by double integration motion in vertical direction can be obtained. In line drag coefficient $\left(\mathrm{C}_{\mathrm{D}}\right)$ can be found by following formula where $F_{D}$ s total in line drag.

$C_{D}=\frac{F_{D}}{0.5 \rho L D U^{2}}$

The length of the path for one cycle is $U / \mathrm{f}_{\mathrm{s}}$, for steady vibration, where $U$ is term used for free stream velocity and $f_{s}$, for the shedding frequency. The width of the path is $2 \mathrm{~A}_{\mathrm{z}}$ where $\mathrm{A}_{\mathrm{z}}$ is the amplitude of the vibration, also can be noted as A .These path dimensions can be related to the structural dimension namely non dimensional motion amplitude (A/D) and reduced velocity $U_{R}$. Normally non dimensional motion amplitude (A/D) is presented as a function of reduced velocity $\left(U_{R}\right)$ to analyze vortex induced vibration [2]. Figure 3 shows spring supported cylinder subjected to current. Where, $\mathrm{U}$ is free stream velocity, $\mathrm{D}$ is cylinder diameter and $f_{n}$ is natural frequency of the cylinder which can be found by following equation

$\mathrm{f}_{\mathrm{n}}=\frac{1}{2 \pi} \sqrt{\frac{\mathrm{k}}{\mathrm{m}+\mathrm{m}_{0}}}$

where $\mathrm{k}$ is spring constant, $\mathrm{m}$ and $\mathrm{m}_{\mathrm{o}}$ are respectively the mass and added mass of the cylinder. When the shedding frequency $\left(f_{s}\right)$ becomes equal to the natural frequency $\left(f_{n}\right)$ of the structure, the lock-in phenomenon occurs. At lock-in, the vibration amplitude becomes maximum and the correlation between the excitation forces along the span increases dramatically. In other words, when the vortex shedding frequency is controlled by the oscillation frequency, lock-in occurs.

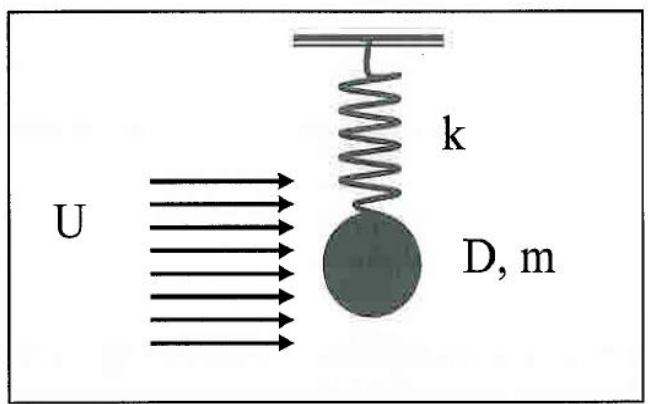

Figure 3 Spring supported cylinder subjected to current

The oscillation frequency will be different than the natural frequency in still water since added mass will be varied. So, it can be said the synchronisation of the shedding and vibration frequencies largely depend on hydrodynamic mass variation. Vikestad [1] showed normally much wider lock-in regions are experienced at low mass ratio of order 1 to 10 . The mass ratio is defined as the ratio of the oscillating structural mass to the displaced fluid mass. For cylinder of length L, it is

$$
\mathrm{m}^{+}=\frac{\mathrm{m}}{\rho \pi \mathrm{L} \frac{\mathrm{D}^{2}}{4}}
$$

\subsection{EXPERIMENTAL SETUP}

Cross flow vibration can be measured by using a towing tank the VIV experiments have been performed in UTM Marine Technology Canter's (MTC) 2.5 x 4 x 120.0 m towing tank, which is equipped with an overhead towing carriage. A circular test cylinder of $114 \mathrm{~mm}$ in diameter and $3 \mathrm{~m}$ in length was suspended from the carriage on two streamlined vertical struts by two pair of springs, two horizontal plates were attached with the end of the pipe as depicted in Figure 4. The cylinder was towed in still water at different velocities to simulate the uniform current condition. For the freely vibrating tests, cylinder was allowed to move up and down whereas to make the cylinder stationary, clamps were used as shown in Figure 5.

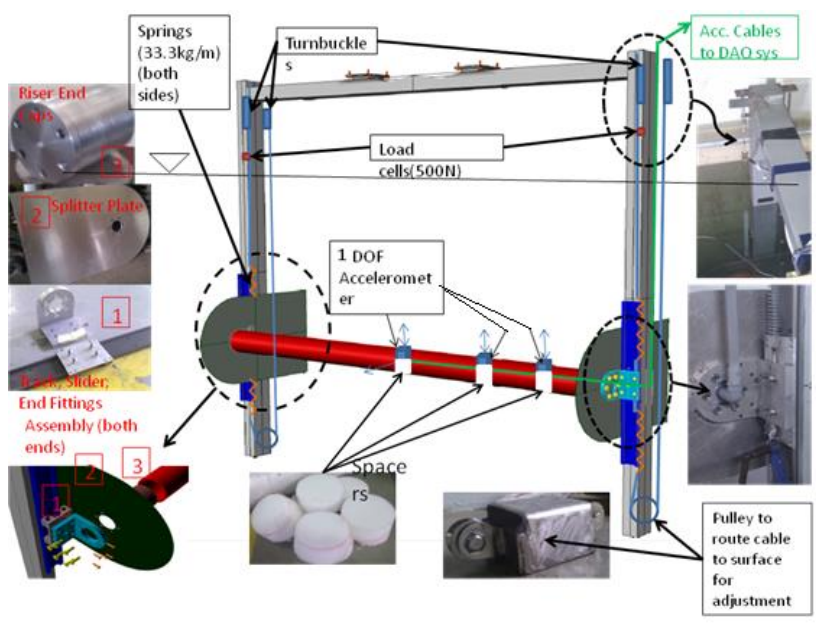

Figure 4 Experimental set up to measure VIV

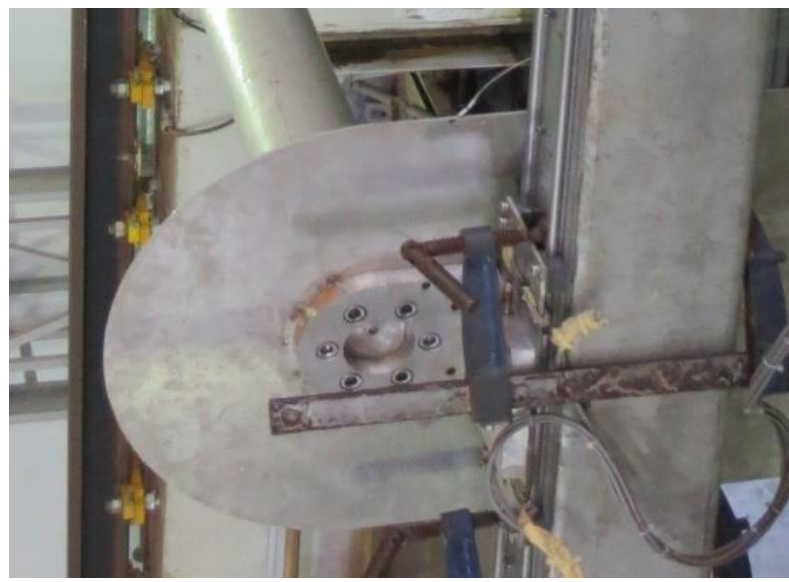

Figure 5 Clamps to make the cylinder stationary

The cylinder was parallel to the free surface and the cylinder was submerged by $1.25 \mathrm{~m}$. The three dimensional flow effects were minimized and local ventilation of the downstream vortices was avoided by using two horizontal plates at the cylinder ends which were about $40 \mathrm{~cm}$ away from the basin wall. The test set up was designed for reduced velocities between $U_{R}=3.5$ and 12, in which upper branch of VIV can be detected [1]. The free surface flow effects and the bottom flow effects were assumed to be negligible. The influence of mechanical vibrations was assumed to be negligible. Only vertical motion was observed through 
accelerometer, in-line drags were observed by strain gauge and lift forces were measured by transducers.

Table 1 Experimental parameters; (Cylinder Particulars)

\begin{tabular}{clccc}
\hline Entry & Parameter & Symbol & unit & Value \\
\hline 1 & Diameter & $\mathrm{D}$ & $\mathrm{mm}$ & 114 \\
2 & Length & $\mathrm{L}$ & $\mathrm{m}$ & 3 \\
3 & Mass ratio & $\mathrm{m}^{+}$ & & 1.18 \\
4 & Natural frequency & $\mathrm{fn}$ & $\mathrm{s}^{-1}$ & 0.70 \\
5 & Aspect Ratio & $\mathrm{L} / \mathrm{D}$ & & 26.31 \\
6 & Damping ratio & $\zeta$ & & 0.01 \\
\hline
\end{tabular}

\subsection{NUMERICAL SIMULATION}

The VIV experiment was tried to be simulated by solving the coupled fluid-structure interaction problem. ANSYS-CFX solver was used for simulations. The coupled fluid-structure interaction problem can be considered as a three field problem, i.e. fluid flow, structural displacement and the moving mesh. By solving fluid flow problem, force on the structure at each time step is calculated then by solving 2 nd order motion equation the structural displacement is calculated. By numerically solving the unsteady incompressible Navier-Strokes equations, the flow flied around the cylinder was calculated, LES was used to solve turbulent flow.

$\frac{\partial \bar{u}_{i}}{\partial t}+\frac{\partial}{\partial x_{j}} \bar{u}_{i} \bar{u}_{j}=-\frac{1}{\rho} \frac{\partial \bar{p}}{\partial x_{j}}+\boldsymbol{v} \frac{\partial \bar{u}_{i}}{\partial x_{i} x_{j}}-\frac{\partial \tau_{i j}}{\partial x_{i}}$

where the sub grid stresses are given by

$\tau_{i j}=\overline{u_{\imath} u_{J}}-\bar{u}_{i} \bar{u}_{j}$

with the smagorinsky sub grid scale turbulence model

$\tau_{i j}=2 v_{T} \bar{S}_{i j}$

$v_{T}=\left(\mathrm{C}_{\mathrm{s}} \Delta\right)^{2} \sqrt{2} \bar{S}_{i j} \bar{S}_{i j}$

The local strain rate tensor $\bar{S}_{i j}$ is defined as

$\bar{S}_{i j}=\frac{1}{2}\left(\frac{\partial \bar{u}_{i}}{\partial x_{j}}+\frac{\partial \bar{u}_{j}}{\partial x_{i}}\right)$

and filter width is taken as the local grid size, for example

$\Delta=\left(\Delta_{\mathrm{x}} \Delta_{\mathrm{y}} \Delta_{\mathrm{z}}\right)^{\frac{1}{3}}$

The Smagorinsky coefficient is taken as 0.1 . The governing equation (Equation 5) for the dynamic response of the rigid horizontal cylinder is discussed earlier. ANSYS-CFX software then solves the governing equation by using CFX Expression Language (CEL) code to determine the cylinder displacement The riser was horizontally positioned as it was during the experiment (see Figure 7).The computational domain in this study extends for 10D in front of the horizontal riser, $30 \mathrm{D}$ behind the horizontal riser, $10 \mathrm{D}$ under and above the horizontal riser, while the breadth of the computational domain remain $\mathrm{L}$ that is $3 \mathrm{~m} .100$ time steps par vortex shedding were employed for this simulation. While doing the mesh the main aim was to provide increased mesh resolution in the wake and near the cylinder and lower resolution in other areas as shown in the Figure 6. This meshing approach was adopted to reduce the total element number and the computational effort. Total number of cells in the mesh for the simulations was 419,202.

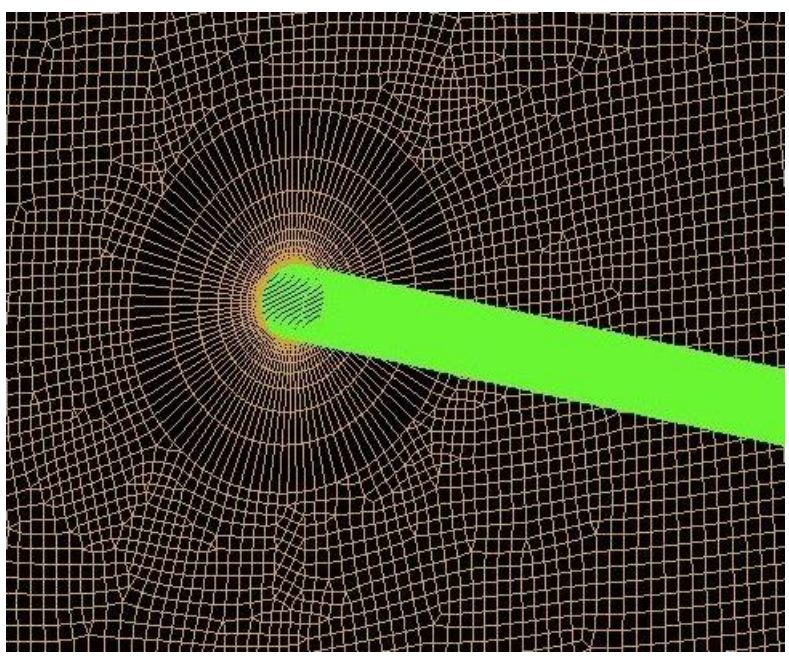

Figure 6 Grid details near boundary layer 


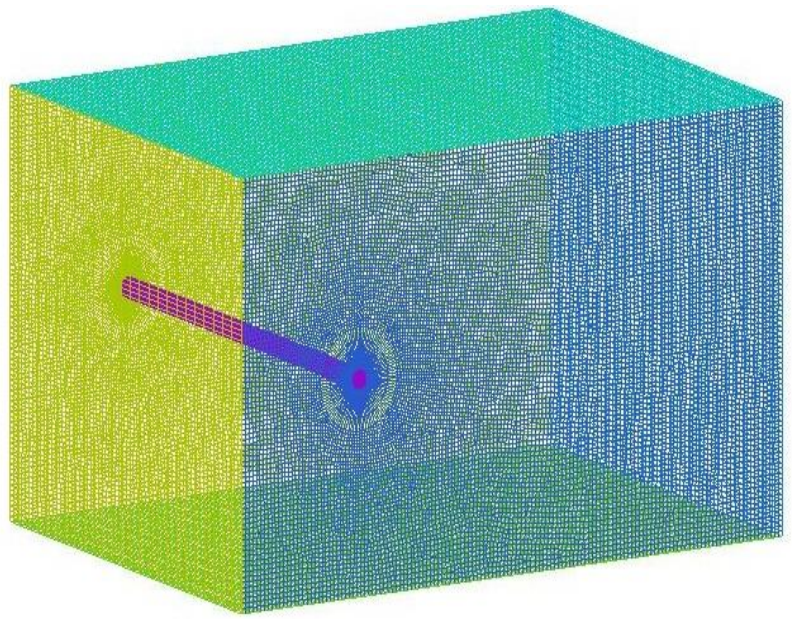

Figure 7 Isometric view of the computational domain

The no-slip boundary condition is imposed on the riser surface and the boundary condition of side walls was set to symmetry .

\subsection{RESULTS AND DISCUSSION}

From Figure 8 to Figure 12, high Reynolds number $\left(\operatorname{Re}<4 \times 10^{5}\right)$ VIV data obtained through UTM experiments are presented along with results calculated through CFD simulations. The results in Figure 8 are showing the ability of Ansys CFX for detecting the oscillating behavior and nearly the same vertical displacement for the cylindrical riser as that for the experimental results at $U_{R}=8$. However, there is a noticeable irregular phase shift between the numerical and the experimental results in the previous figure which is due to sensitivity of the vertical riser displacement to the free stream flow condition, where the uniform flow condition was imposed exactly at the inlet boundary condition at all CFD simulations in this study, while in the experimental tests in UTM towing tank this uniform flow condition cannot be attained exactly. In addition, this miss matching between the experimental and the numerical results can also be attributed to the inability of the turbulence models even with the use of LES for predicting precisely the complex flow pattern of VIV for such cases [13]. At Figure 9 UTM VIV data of non dimensional motion amplitude (A/D) has been compared with CFD results. The oscillation amplitudes in UTM experiments follow the well known "bell shape curve", with a maximum amplitude over diameter value (A/D) of 1.19 at a reduced velocity of $\mathrm{U}_{\mathrm{R}}=6.2$, though the amplitude over diameter value remain near 1 from reduced velocity 6 to 8.5. Results from CFD shows maximum amplitude over diameter value $(A / D)$ occurred at a reduced velocity of $U_{R}=$ 6.5. The trend of these two curves are similar though magnitude of non dimensional motion amplitudes obtained through CFD are higher than that of the experiment results bellow $U_{R}=5$. Peak values from $\mathrm{CFD}$ are lower compared to peak values from experiment.

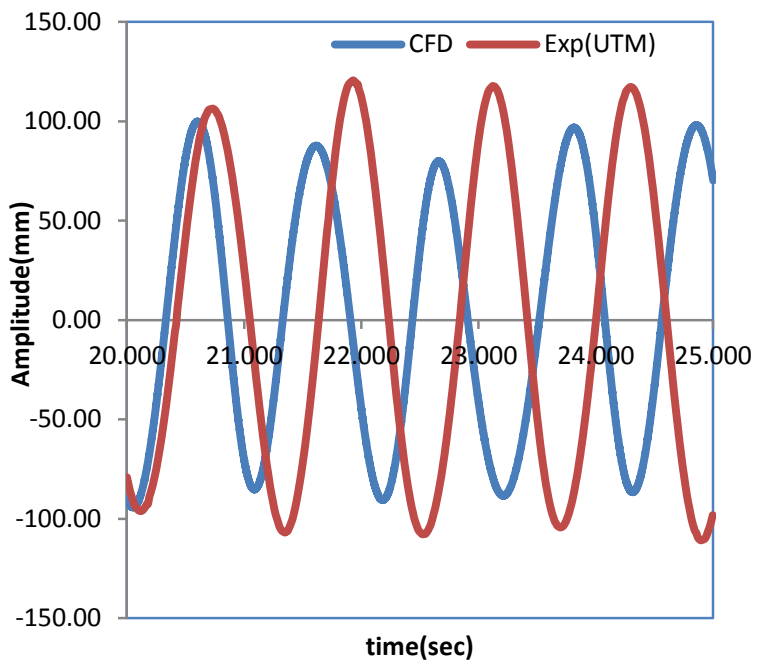

Figure 8 Vibration amplitude vs Time at $\mathrm{U}_{\mathrm{R}}=8$

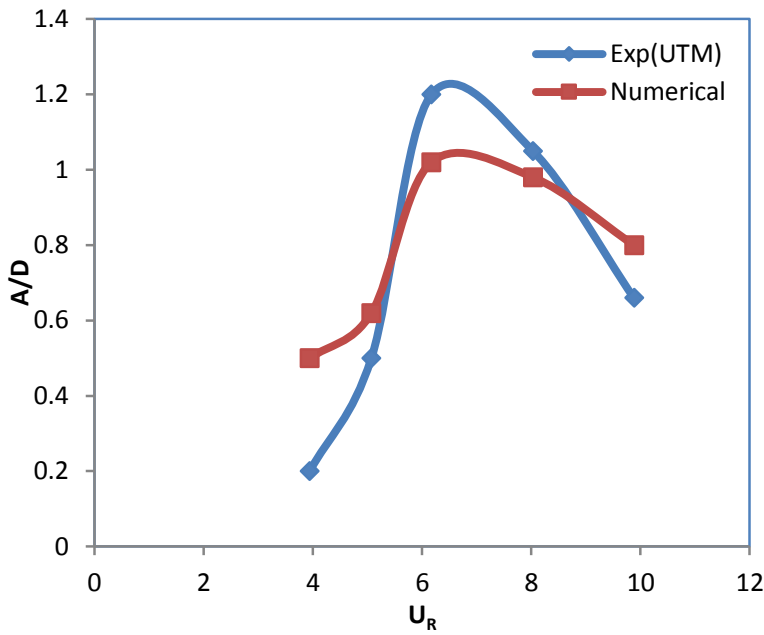

Figure 9 A/D plotted as a function of $U_{R}$

At lower reduced velocity, CFD over predicts the VIV amplitude though the lift coefficients calculated from CFD are lower at low reduced velocity compared to lift coefficients observed at experiment as shown in Figure 10. This anomaly may be occurred due to under prediction of added mass by CFD at lower reduced velocity as shown in Figure 12. In experiment negative added mass was detected at $U_{R}=10$, which is due to the phase between motion and hydrodynamic force. 


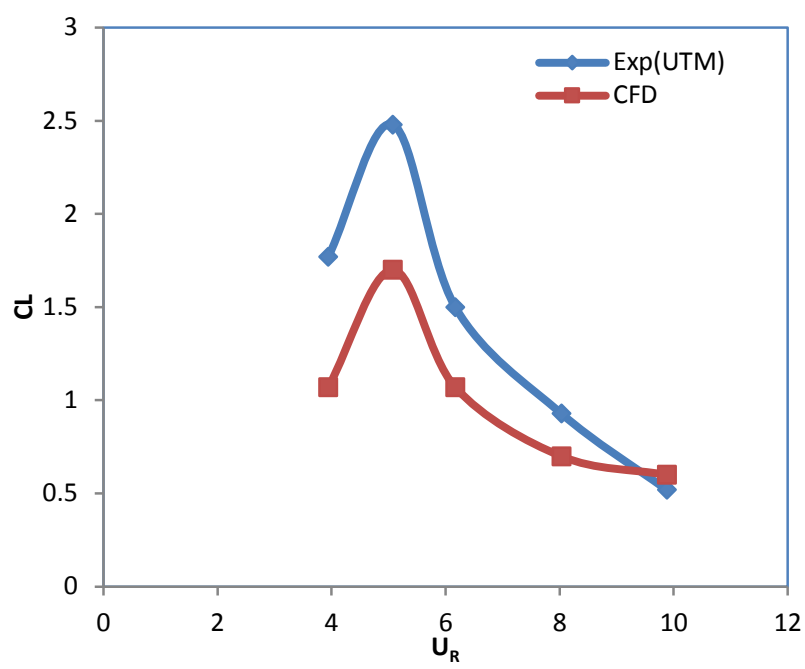

Figure $10 \mathrm{CL}(\mathrm{rms})$ plotted as a function of $\mathrm{U}_{\mathrm{R}}$

From Figure 11, it can be seen that the maximum in-line drag coefficient occurs at small reduced velocities. For higher reduced velocities $\left(U_{R}>6\right)$, a clear decrease in in-line drag coefficient can be observed. The maximum in-line drag coefficient from experiment is approximately 2.5 found at $U_{R}=6$. Shape of the inline drag coefficient curve obtained through CFD is in accordance with the curve found by UTM test though the magnitudes' of CD are bit higher than that of experiment. The flow pattern around the cylinder is depicted in Figure 13 and Figure 14. The development of wake turbulence after laminar separation is evident.

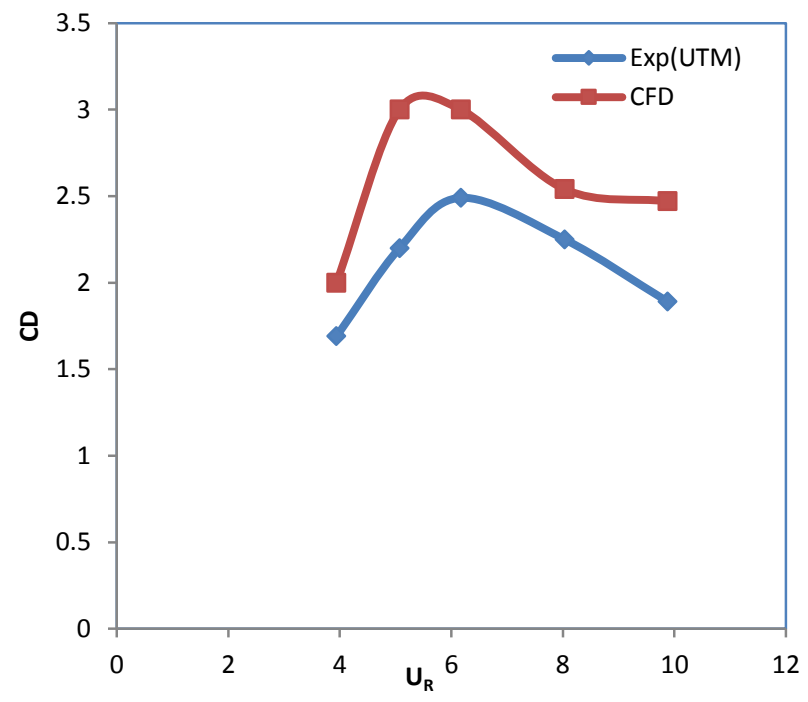

Figure $11 C_{D}$ (mean) plotted as a function of $U_{R}$

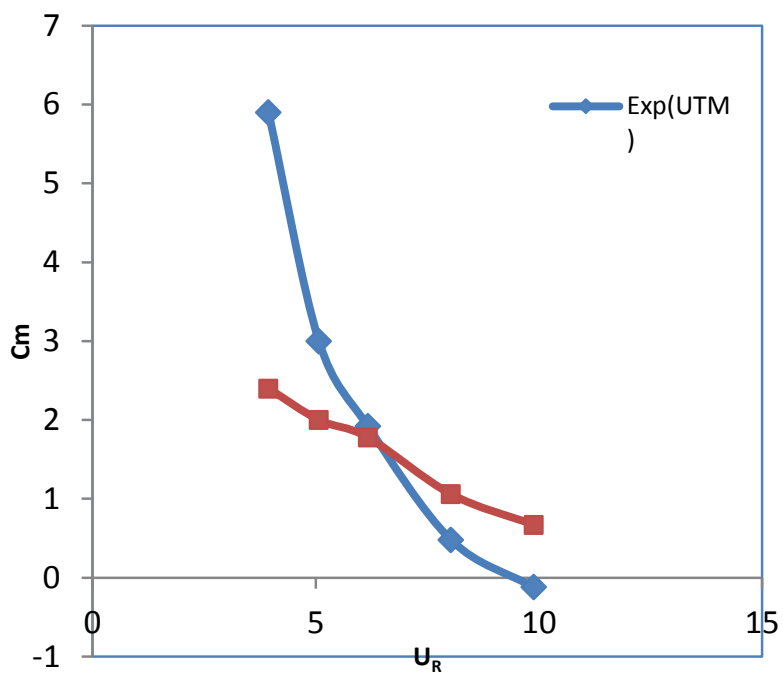

Figure $12 \mathrm{Cm}$ (added mass coefficient ) plotted as a function of $U_{R}$

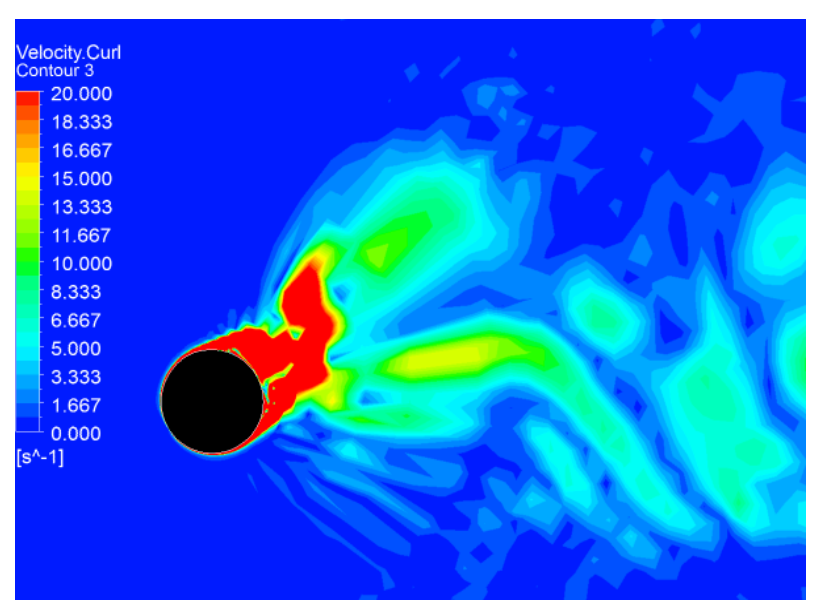

Figure 13 velocity curl at $\mathrm{U}_{\mathrm{R}}=8$ at $21 \mathrm{sec}$

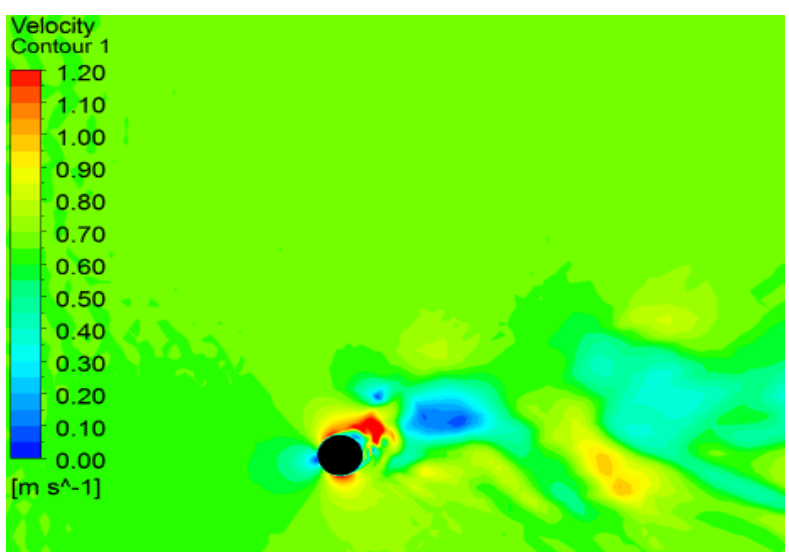

Figure 14 Velocity contour at $U_{R}=8$ at $21 \mathrm{sec}$

At a certain speed, the vibrations of the cylinder cause higher drag loads than for the same cylinder kept stationary, which is termed as "drag amplification". The maximum drag amplification factor in UTM tests is about 2.5 that was found at lower reduced velocity $\mathrm{U}_{\mathrm{R}}=6$. At Figure 15, UTM drag amplification factors are 
compared with drag amplification factors found from CFD. CFD values are higher than experiments ones with a maximum value of 3.75 at $U_{R}=6$.

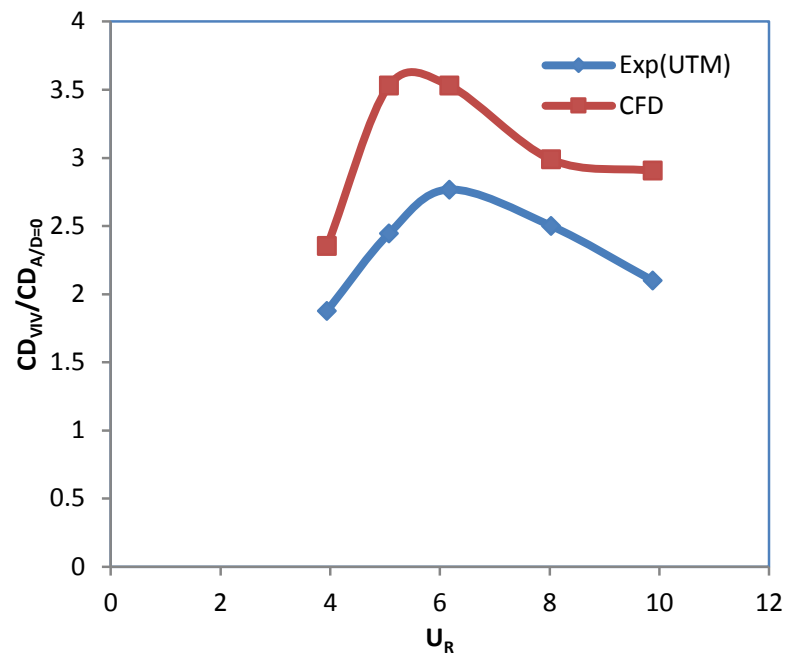

Figure 15 Ratio between in-line drag coefficient $C D$ VIV and $C D D_{A I D=O}$

\subsection{CONCLUSION}

Details of the test set-up that was used for VIV measurements of a cylinder with low mass ratio at high Reynolds numbers are presented. CFD was used to obtain VIV data to compare with the experiment results. Results from 3D simulation of VIV by using LES turbulence model show reasonably good agreement with experimental results. The VIV amplitudes obtained through experiments are in agreement with the experience of similar kind of tests done by previous researchers in terms of achieving a clear "bell shaped" curve. Peak values are observed in between reduced velocities of 6 and 8.5 and recorded maximum A/D-value approximately 1.19. However the VIV amplitudes obtained through CFD have little lower peak value than that of experiment. The discrepancies between experiments and simulations results can be attributed to the mesh fine-tuning and turbulence model. CFD results of this paper are significant as majority of VIV simulations found from literatures dealt with 2D flows and lower Reynolds numbers. 3D numerical simulation can be very handy to determine the effectiveness of different VIV suppression devices.
Details of experiment setup mentioned in this paper also can be very helpful for similar kind of researches in future.

\section{Acknowledgement}

We are grateful for the UTM scholarship to Author 1 and UTM research grant $(02 \mathrm{H} 51)$ for funding this project.

\section{References}

[1] Vikestad, K. 1998. Multi-Frequency Response of a Cylinder Subjected Vortex-Shedding and Supported Motions. D.Sc. thesis, Department of Marine Structures, Norwegian University of Science and Technology, Trondheim, Norway.

[2] Blevins, R. D. 1990. Flow-Induced Vibrations. 2 Edition. Van Nostrand Reinhold. ISBN 0-442-20651-B.

[3] Govardhan, R. Williamson, C. H. K. 2000. Modes of Vortex Formation and Frequency Response of a Freely Vibrating Cylinder. Journal of Fluids Mechanics. 420: 85-130.

[4] Willden, R. H. J., Graham, J. M. R. 2004. Multi-modal Vortex-Induced Vibrations of a Vertical Riser Pipe Subject to a Uniform Current Profile. European Journal of Mechanics B/Fluids. 23: 209-218.

[5] Dong, S., and Karniadakis, G. E. 2005. DNS of Flow past a Stationary and Oscillating Cylinder at Re D 1000. J Fluids and Struct. 20: 519-531.

[6] Dong, S., Karniadakis, G. E., Ekmekci, A, and Rockwell, D. 2006. A Combined Direct Numerical Simulation-Particle Image Velocimetry Study of the Turbulent Near Wake. J Fluid Mech. 569: 185-207.

[7] Chaplin, J. R., Bearman, P. W., Cheng, Y., Fontaine, E., Graham, J. M. R., Herfjord, K., Huera, F. J., Isherwood, M., Lambrakos, K., Larsen, C. M., Meneghini, J. R., Moe, G., Pattenden, R. J., Triantafyllou, M. S., Willden, R. H. J. 2005. Blind Prediction of Laboratory Measurements of Vortex-Induced Vibrations of a Tension Riser. Journal of Fluids and Structures. 21: 25-40.

[8] Trim, A. D., Braaten, H., Lie, H., Tognarelli, M. A. 2005. Experimental Investigation of Vortex-induced Vibration of Long Marine Risers. Journal of Fluids and Structures. 21: 335-361.

[9] Lucor, D. Mukundan, H, Triantafyllou, M. S. 2006. Riser Modal Identification in CFD and Full-scale Experiments. Journal of Fluids and Structures. 22: 905-917.

[10] Holmes, S., Oakley, O.H., Constantinides, H. 2006. Simulation of Riser VIV Using Fully Three Dimensional CFD Simulations. OMAE 200692124, 25th International Conference on Offshore Mechanics and Artic Engineering, Hamburg, Germany.

[11] Constantinescu, G., Pacheco, R., Squires, K. D. 2002 Detached-Eddy Simulation of flow over a sphere. AIAA Paper 2002-0425.

[12] Constantinescu, G., Chapelet, M., Squires, K. D. 2003. On Turbulence Modeling Applied to Flow Over a Sphere. AIAA Journal. 41: 1733-1742.

[13] F. Saltara, A. D. Agostini Nato, J. I. Z Lopez. 2011. 3D CFD Simulation of Vortex-induced Vibration of Cylinder. International Journal of Offshore and Polar Engineering. 21(3): 192-197. 